\title{
Expression analysis of the Arabidopsis thaliana AtSpen2 gene, and its relationship with other plant genes encoding Spen proteins
}

\author{
María Gloria Solís-Guzmán ${ }^{1}$, Gerardo Argüello-Astorga ${ }^{2}$, José López-Bucio ${ }^{1}$, León Francisco Ruiz-Herrera ${ }^{1}$, \\ Joel López-Meza $^{1}$, Lenin Sánchez-Calderón ${ }^{3}$, Yazmín Carreón-Abud ${ }^{1}$ and Miguel Martínez-Trujillo ${ }^{1}$. \\ ${ }^{1}$ Universidad Michoacana de San Nicolás de Hidalgo, Morelia, Michoacán. Mexico. \\ ${ }^{2}$ Instituto Potosino de Investigación Científica y Tecnológica, San Luis, S.L.P., Mexico. \\ ${ }^{3}$ Universidad Autónoma de Zacatecas. Zacatecas, Zac., Mexico.
}

\begin{abstract}
Proteins of the Split ends (Spen) family are characterized by an N-terminal domain, with one or more RNA recognition motifs and a SPOC domain. In Arabidopsis thaliana, the Spen protein FPA is involved in the control of flowering time as a component of an autonomous pathway independent of photoperiod. The $A$. thaliana genome encodes another gene for a putative Spen protein at the locus At4g12640, herein named AtSpen2. Bioinformatics analysis of the AtSPEN2 SPOC domain revealed low sequence similarity with the FPA SPOC domain, which was markedly lower than that found in other Spen proteins from unrelated plant species. To provide experimental information about the function of AtSpen2, A. thaliana plants were transformed with gene constructs of its promoter region with uidA::gfp reporter genes; the expression was observed in vascular tissues of leaves and roots, as well as in ovules and developing embryos. There was absence of a notable phenotype in knockout and overexpressing lines, suggesting that its function in plants might be specific to certain endogenous or environmental conditions. Our results suggest that the function of Atspen2 diverged from that of fpa due in part to their different transcription expression pattern and divergence of the regulatory SPOC domain.
\end{abstract}

Keywords: Spen; Arabidopsis; vascular bundle; expression.

Received: August 15, 2016; Accepted: March 30, 2017.

\section{Introduction}

Several experimental approaches have been used to determine the function of genes in plants: inactivation by disruption (knockout), overexpression using strong or constitutive promoters, single gene or whole transcriptome analysis, and the use of reporter constructs to study expression patterns conferred by regulatory regions. Notwithstanding the variety of existing methods, only approximately $25 \%$ of the genes of Arabidopsis thaliana, the most-studied plant species, have been functionally characterized (Rhee and Mutwil, 2014). Using different databases, it is possible to determine the presence of potential cis elements in the promoters of genes under study, and predict if transcription is developmentally regulated or if it is regulated by specific internal or external factors (Hernández-García and Finer, 2014).

With the growing number of sequenced plant genomes, numerous databases have been established that can be

Send correspondence to Miguel Martínez-Trujillo, Laboratorio de Genética y Microbiología. Facultad de Biología, Universidad Michoacana de San Nicolás de Hidalgo, Francisco J. Múgica s/n, Col. Felícitas del Río, 58030 Morelia, Michoacán, Mexico. E-mail: codigogenetico@gmail.com explored by using various bioinformatics tools to predict the functions of genes and their products. From a structural and evolutionary point of view, it has been possible to classify proteins into families, mainly by comparing related sequences and domains and the presence of tertiary structures associated with specific functions that have been preserved during molecular evolution (Martínez, 2011). The current definition of more than 10,000 protein families could be considered, by analogy, as a periodic table for biology, allowing the prediction of protein functions at the molecular level (Sammut et al., 2008). By means of diverse algorithms, protein domains can be predicted from the amino acid sequence to obtain the first clues about molecular function (Marchler-Bauer et al., 2015).

Proteins of the Split ends (Spen) family are characterized by having an N-terminal RRM domain (RNA recognition motif) and a C-terminal conserved SPOC domain (Spen paralogue and orthologue C-terminal) (Kuang et al., 2000). The RRM domain is also known as an RBD (RNA binding domain) or an RNP (Ribonucleoprotein domain); it consists of 90 amino acids and has a structure composed of four beta sheets and two alpha helices that interact with ssDNA and ssRNA. This domain is found in a variety of 
hnRNPs (heterogeneous nuclear ribonucleoproteins) involved in alternative splicing and in proteins that are components of snRNPs (small nuclear ribonucleoproteins) (Ariyoshi and Schwabe, 2003). The SPOC domain contains approximately 165 amino acid residues and consists of a beta-barrel with seven sheets $(\beta 1-\beta 7)$ framed by six alpha helices $(\alpha \mathrm{A}-\alpha \mathrm{F})$ and is involved in protein-protein interactions; this domain has been described among animals, plants and fungi (Sánchez-Pulido et al., 2004).

The Spen proteins of animals are involved in different biological processes. In Drosophila, they participate in neuronal cell differentiation, the growth and guidance of axons in the embryo (Chen and Rebay, 2000; Kuang et al., 2000), cell-cycle regulation (Lane et al., 2000), and repression of head identity of the embryonic trunk (Wiellette et al., 1999). Artavanis-Tsakonas et al. (1999) stated that the Notch/RBP-Jkappa signaling pathway is involved in several cellular processes during the embryonary development, such as proliferation, differentiation, apoptosis, maintenance of undifferentiated cells, and cell-fate specification. In humans, the Spen SHARP protein (SMRT7/HDAC1-associated repressor protein) participates as a component of this signaling pathway, repressing the transcription of the Notch genes by the RBP-Jk-SHARP complex, which recruits a histone deacetylase complex (Oswald et al., 2002). Furthermore, the participation of SHARP in the silencing of the $\mathrm{X}$ chromosome in humans has been recently demonstrated: the long non-coding RNA Xist associates with SHARP and two other proteins (SAF-A and LBR), forming a complex that activates a histone deacetylase (DHAC3) which is necessary not only for silencing the chromosome but also for the exclusion of the RNA polymerase II (McHugh et al., 2015).

Flowering is a process that entails the change from the vegetative to the reproductive phase in the angiosperms; it depends on the quantitative integration of environmental stimuli with an endogenous development program (Simpson and Dean, 2002). In A. thaliana several flowering pathways have been characterized, including the photoperiod-dependent pathway, the gibberellin (GA) pathway, and the autonomous pathway, which is not triggered by light signals but by exposure to low temperatures in combination with some endogenous cues (Simpson, 2004). In the autonomous pathway, the components act regulating the level of the mRNA that encodes the Flowering Locus C (FLC), a transcription factor that repress flowering by antagonizing the activity of some components of the photoperiod-dependent pathway (Hepworth et al., 2002). The $A$. thaliana Spen protein FPA (Flowering Pathway Autonomous) is a key component of the autonomous pathway (Schomburg et al., 2001). It acts in concert with other proteins (FCA and FY) promoting the 3' end processing and polyadenylation of an antisense flc RNA (Hornyik et al., 2010), leading to the transcriptional downregulation of the sense flc RNA, by a mechanism involving the FLD demethylase (Liu et al., 2010). Thus, the flowering time in A. thaliana is increased or reduced when the fpa gene is either inactivated or overexpressed, respectively (Schomburg et al., 2001).

The crystal structure of the FPA SPOC domain has been determined at high resolution $(2.7 \AA)$ and comparisons with homologous domains showed the highest similarity with the SPOC domain of SHARP, hence demonstrating the high conservation of the SPOC structure among far related organisms (Zhang et al., 2016).

In rice (Oryza sativa), two genes encoding Spen proteins, OsRRM and OsRRMh, have been identified. OsRRM is expressed in the seed endosperm and the synthesized protein is localized in the nucleus. However, when this gene was silenced by dsRNAi, no differences with the control plants were found in the seeds and whole plant phenotypes, and no change in the flowering time was observed (Chen et al., 2007). The OsRRMh gene is expressed in the roots, stem, leaves, and immature seeds, and it is alternatively spliced in different tissues, suggesting its involvement in several biological functions. When this gene was knocked down by dsRNAi, the flowering was retarded and the panicle phenotype was larger, which was in agreement with the down-regulation of two flowering-related genes of rice: RFT1 and Hd3a (Liu and Cai, 2013).

With the sequencing of the $A$. thaliana genome (Arabidopsis Genome Initiative, 2000), it has been possible to identify numerous putative genes, but the functions of many remain enigmatic. In this work, experimental and bioinformatics information was generated and analyzed to unravel the function of the At4g12640 gene (herein named AtSpen2) encoding a putative Spen protein (AtSPEN2). Gene constructs of the AtSpen 2 promoter fused to uidA::gfp reporter genes revealed its expression in the vascular tissues of leaves, roots, and embryos, while no apparent changes in growth, development and flowering time were observed under standard growth conditions in AtSpen 2 knockout (KO) mutants or overexpressing (OE) lines compared to WT plants. We discarded that AtSpen 2 has a function related with the flowering time, and suggest that it may have an accessory function in a development process or its participation is conditional and depends on certain endogenous or environmental conditions.

\section{Materials and Methods}

\section{Similarity analysis of the AtSPEN2 SPOC domain}

Sequences were obtained from http://www.ncbi.nlm.nih.gov and http://www.uniprot.org. Multiple sequence alignment was performed with ClustalW. The tree was generated with Mega 6.0 (Tamura et al., 2013), using the Neighbor-Joining method with the proportion of paired differences. 


\section{Construction of a vector to overexpress AtSpen2}

A region of the AtSpen 2 cDNA was amplified from a plasmid isolated from an $A$. thaliana cDNA Matchmaker ${ }^{\circledR}$ library. The oligonucleotides FOE 5'-AAAAAGCAGGCT GCATGTCATCTAGAGGAAGGGAGAGGATGA-3' and ROE 5'-AGAAAGCTGGGTGTTAACTCGGTTT AGCTTGTTGAATCTGCTG-3' were used to generate the $a t t B 1$ and $a t t B 2$ recombination sites. The amplified DNA fragment was cloned into the pDONR22 $1^{\circledR}$ (Invitrogen) vector, harboring the sites attP1 and attP2. The recombination reaction was performed using the BP Clonase Enzyme Mix Kit ${ }^{\mathbb{B}}$ (Invitrogen), forming the attL1 and attL2 recombination sites. The pDONR $221{ }^{\circledR}$ vector with the AtSpen 2 coding region was recombined with the pK2GW7.0 (Ghent University, Belgium) binary vector (harboring the attRl and $a t t R 2$ recombination sites), using the LR Clonase Enzyme Mix Kit ${ }^{\circledR}$ (Invitrogen). In the final recombinant vector, the strong 35S promoter of the Cauliflower Mosaic Virus (Odell et al., 1985) was joined to the AtSpen 2 coding region.

\section{Genotyping of the AtSpen2 knockout mutant (KO)}

Seeds of the $A$. thaliana insertional mutant At4g12640-T-DNA (KO) were provided by the Salk Institute for Genomic Analysis. Two lines were used: KO-024223 and KO-030932. Lines were genotyped by PCR as previously described (Alonso et al., 2003).

\section{Arabidopsis thaliana genomic DNA extraction and amplification of the promoter region}

DNA of the wild-type $A$. thaliana ecotype Col-0 was isolated using the DNAeasy Plant Mini Kit (QIAGEN), following the manufacturer's instructions. The resulting DNA was used to amplify $500 \mathrm{bp}$ of the AtSpen 2 promoter region, using the oligonucleotides: F500 5'-ACATGACGAGCA GATCTACGGAGA-3' and R500 5'-TCTGCATTCGTC AGATCTATCGCA-3'. The Supermix High Fidelity ${ }^{\circledR}$ PCR kit (Invitrogen) was used following the manufacturer's instructions. The amplification conditions were $95^{\circ} \mathrm{C}$ for $5 \mathrm{~min}, 30$ cycles of $95{ }^{\circ} \mathrm{C}$ for $1 \mathrm{~min}, 55^{\circ} \mathrm{C}$ for 1 min, $68^{\circ} \mathrm{C}$ for $90 \mathrm{~s}$, followed by a final extension at $68^{\circ} \mathrm{C}$ for $10 \mathrm{~min}$.

\section{Construction of a vector to analyze the expression conferred by the AtSpen2 promoter region}

The $500 \mathrm{bp}$ PCR fragment, corresponding to the AtSpen 2 promoter, was cloned into the $\mathrm{pCR} 8^{\circledR} / \mathrm{GW} / \mathrm{TOPO}$ (Invitrogen) vector, between the attL1 and attL2 recombination sites. The sequence and orientation of the cloned fragment was verified by the method of Sanger et al. (1977). The resultant vector was recombined with the pKGWFS7 binary vector (Ghent University, Belgium) containing the attR1 and attR2 recombination sites. This vector harbors a translational fusion of the $g f p$ and $u i d A$ reporter genes, allowing both to be used for analyzing the ex- pression conferred by the AtSpen 2 promoter region. Recombination was performed using the LR Clonase II enzyme Mix ${ }^{\mathbb{B}}$ Kit (Invitrogen).

\section{Agrobacterium tumefaciens and Arabidopsis thaliana genetic transformation}

The recombined binary vectors were used to transform the Agrobacterium tumefaciens strain pGV2260 (McBride and Summerfelt, 1990) by electroporation at $1800 \mathrm{~V}$, using the Eppendorf electroporator 2510. Transformed colonies were selected in LB medium (Luria) with carbenicillin $(100 \mu \mathrm{L} / \mathrm{mL})$, rifampicin $(50 \mu \mathrm{L} / \mathrm{mL})$, spectinomycin $(100 \mu \mathrm{L} / \mathrm{mL})$ and streptomycin (300 $\mu \mathrm{L} / \mathrm{mL})$.

The A. tumefaciens strain pGV2260 containing the recombinant binary vector was grown to an absorbance of $0.6(600 \mathrm{~nm})$, centrifuged for $5 \mathrm{~min}$ at $4500 \mathrm{~g}$. The pellet was resuspended in infiltration medium: 0.5X MS (Murashige and Skoog, 1962), 5\% sucrose (Bioxon) and 0.05\% Silwett L-77. The bacterial suspension was applied to $2-10$ $\mathrm{mm}$ A. thaliana Col-0 inflorescences following the floral DIP method with some modifications (Clough and Bent, 1998; Martínez-Trujillo et al., 2004). Plants were placed in the dark for $12 \mathrm{~h}$ and then transferred to light conditions. To collect the seeds, plants were allowed to produce mature siliques. Plant selection was made in $0.2 \mathrm{X}$ MS medium, $\mathrm{pH}$ 5.7 supplemented with $0.6 \%$ sucrose as carbon source and $1 \%$ plant agar (Phytotechnology Laboratories A111), with kanamycin $(60 \mu \mathrm{g} / \mathrm{mL})$ for plant selection.

\section{Expression analysis by real-time quantitative PCR (RT-qPCR)}

Total RNA from Col-0, KO- and OE A. thaliana lines was isolated from the roots, leaves, and flowers, using Trizol $^{\circledR}$ (Invitrogen). cDNA synthesis was performed using reverse transcriptase $\mathrm{H}$ (Thermo Fisher Scientific) and oligo dT. The following oligonucleotides were designed to amplify a 201 bp AtSpen 2 gene fragment: FAt4g 5'-TCCAAAGGGACTCCAGAATG-3' and RAt4g 5'-CATAACTGCGACCAGGGAAT-3'. A 226 bp fragment of actin was used as the internal reference, using the oligonucleotides FACTINA 5'-TGCCAATCTACGAGG GTTTC-3', RACTINA 5'-TTCCGATGGAAGAGCT GGT-3'.

\section{Growth conditions of different $A$. thaliana lines}

Seeds of the different $A$. thaliana lines were surface-disinfected by washing with $96 \%$ ethanol (v/v in water) for $7 \mathrm{~min}$ and then with $20 \%$ sodium hypochlorite $(\mathrm{v} / \mathrm{v}$ in water) for another $7 \mathrm{~min}$. Subsequently, seeds were rinsed five times with sterile deionized water, resuspended in $1 \mathrm{~mL}$, and placed at $4{ }^{\circ} \mathrm{C}$ in the dark for $48 \mathrm{~h}$ to promote and synchronize germination.

Disinfected seeds were placed in Petri dishes containing 0.2X MS medium, $\mathrm{pH}$ 7.0, for plant growth, supple- 
mented with $0.6 \%$ sucrose and $1 \%$ plant agar. For the selection of genetically transformed plants, kanamycin (60 $\mu \mathrm{g} / \mathrm{mL}$ ) was added to the medium. Ten days after germination, plants were transferred to pots with a substrate composed of peat moss, perlite, and vermiculite in 3:1:1 ratios at field capacity water. Both Petri dishes and pots were incubated in growth chambers (Percival Scientific AR-95L), under the following conditions: $24{ }^{\circ} \mathrm{C}, 100 \mu \mathrm{mol} \mathrm{m} \mathrm{s}^{-1}$ light intensity, $80 \%$ relative humidity, and a $16: 8 \mathrm{~h}$ light-dark photoperiod.

\section{Expression analysis of the uidA and gfp reporter genes}

The transgenic plants with the uidA reporter gene (Jefferson et al., 1987) were stained with $0.1 \%$ 5-bromo-4cloro-3-indolyl D-glucuronide (x-gluc) in phosphate buffer $\left(\mathrm{NaH}_{2} \mathrm{PO}_{4}\right.$ and $\mathrm{Na}_{2} \mathrm{HPO}_{4}, 0.1 \mathrm{M}, \mathrm{pH}$ 7), with $4 \mathrm{mM}$ potassium ferrocyanide and potassium ferricyanide for $12 \mathrm{~h}$ at $37^{\circ} \mathrm{C}$. A blue compound was generated by conversion of the $\mathrm{x}$-gluc substrate via the activity of the beta-glucuronidase enzyme encoded by uidA. Plants were clarified and fixed according to Malamy and Benfey (1997).

The expression of the $g f p$ reporter gene was determined by detecting the green fluorescence emitted by the GFP protein (Haseloff and Amos, 1995). To analyze the expression pattern, seedlings were supplied with propidium iodide $(1 \mu \mathrm{g} / \mathrm{mL})$, which emits red fluorescence. Detection of GFP and propidium iodide fluorescence was performed using an Olympus Fluo-View FV1000-PME microscope. To generate the fluorescence of the GFP protein and propidium iodide, excitation wavelengths of $488 \mathrm{~nm}$ and 493 $\mathrm{nm}$ were used, respectively; photographs were taken at 509 $\mathrm{nm}$ and $535 \mathrm{~nm}$ wavelengths, respectively.

\section{Results}

\section{Bioinformatics analysis of the AtSpen2 gene and the putative AtSPEN2 protein}

To provide information about the function of the AtSpen 2 gene, the similarity of the encoded protein with other Spen proteins of plants and particularly of the SPOC domain was performed by a bioinformatics analysis.

The AtSpen 2 gene is located on A. thaliana chromosome 4 and encodes a putative protein of 823 amino acid (aa) residues. A bioinformatics analysis of this protein (http://www.ncbi.nlm.nih.gov/protein/42566726) predicted two RRM motifs between amino acids 25-90 and 153-223, respectively, and a SPOC domain from aa residues 471-567 (Figure 1A). Hence, it can be concluded that this protein belongs to the Spen family (Sammut et al., 2008). The alignment of the SPOC domain of AtSPEN2 with the homologous domains of other Spen proteins showed the conservation of 18 amino acid residues, and the sequences that participate in the $\beta$ sheets and $\alpha$ helix structures (Figure 1B). A topological comparison of the RRM and SPOC domains of AtSPEN2 with other described Spen proteins showed that the number of actual or putative RRM domains is either two (AtSpen2, OsRRM and OsRRMh), three (AtFPA) or four (SHARP), but there is only one SPOC domain in the carboxyl side of the protein (Figure 1C).

The average size of plant Spen proteins is approximately 900 aa, while the human protein SHARP is almost four times larger, with 3,664 aa. Unlike the RRM domain, which is very diverse (Maris et al., 2005), the SPOC domain is more conserved, allowing evolutionary relationships to be established between proteins. The bioinformatics analysis of SPOC domains in predicted proteins of vascular plants with a sequenced genome revealed that in each plant species there are two genes encoding Spen proteins. Moreover, the SPOC domains of those distinct proteins encoded in the genome of each plant species are grouped in two clearly divergent clades (Figure 2), hence suggesting that the genes encoding Spen proteins of a same clade are orthologous. This also indicates that the Spen genes of vascular plants were derived from an antique event of duplication of a single ancestral gene. This supposition is reinforced by the existence of only one Spen gene in the non-vascular plant Physcomitrella patents, a moss with a sequenced genome. The moss Spen protein contains a SPOC domain, which displays low sequence identity with both of the major SPOC clades of vascular plants (Figure 2). The comparative analysis of SPOC domains of $A$. thaliana and Oryza sativa proteins showed that the domains of AtSPEN2 and OsRRM belong to the same clade, whereas the domains of FPA and OsRRMh, both reportedly involved in flowering time determination in the corresponding plant species, belong to the second major clade (Figure 2).

\section{Analysis of the AtSpen2 overexpressing (OE) and knockout (KO) transgenic lines}

The binary vector with the AtSpen 2 cDNA fused to the strong $35 \mathrm{~S}$ promoter of Cauliflower mosaic virus (CaMV) was used to transform $A$. thaliana plants using $A$. tumefaciens. Plants were selected based on kanamycin resistance; homozygous plants were selected. This strategy obtained 13 lines (T3 generation). The AtSpen $2 \mathrm{KO}$ lines were analyzed by PCR and it was found that in KO-024223 the T-DNA was inserted in the exon 3 (nucleotide +2153 ), whereas in the KO-030932 line the T-DNA was inserted in the promoter (nucleotide - 413). These KO lines are named $\mathrm{KO}-\mathrm{E}$ and $\mathrm{KO}-\mathrm{P}$ in the following sections.

The in vitro growth of plants from three $\mathrm{OE}$ lines (OE-1, OE-2, OE-3) and two KO lines (KO-E and KO-P), was compared to the WT for 10 days, and then the plants were transferred to pots for the production of flowers and siliques. The following phenotypic variables were analyzed: primary root growth, lateral root number, lateral root density, root biomass, shoot biomass, and flowering time. 


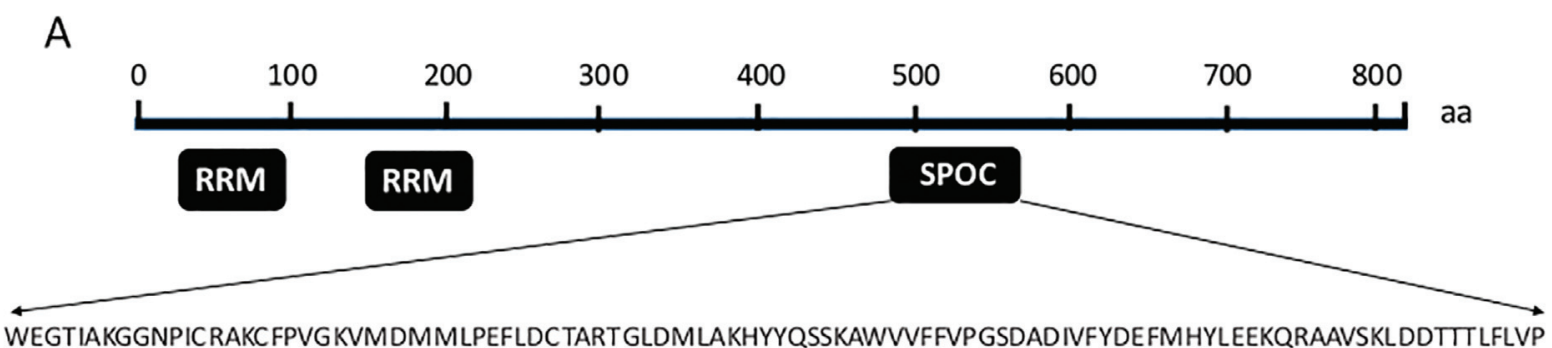

B

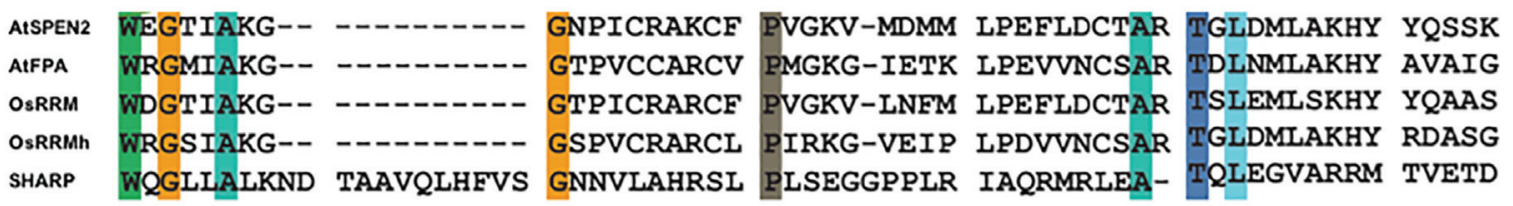

$\beta$

$\beta$

$\begin{array}{lll}\alpha & \beta & \beta\end{array}$

$\alpha$

$\begin{array}{llll}\text { AISPEN2 } & \text { AWVVF FVP--GSDAD IVFYDE---- } \\ \text { AtFPA } & \text { CEIVF FVP--DREED FASYTE---- } & \text { FLRYLSSKD RAKQ RAAVSKLDD- TTT-----LF LVP } \\ \text { OSRRM } & \text { SWVVF FVP--ENDAD MAAYNE---- -FMNYLGDKQ RAAVCKLGE- RSS-----LF LVP } \\ \text { OSRRMh } & \text { FDIVF FLP--DSEDD FVSYTE---- -FLRYLGSKS RAGVVKVDG- GTT-----LF LVP } \\ \text { SHARP } & \text { YCLLL ALPCGRDQED VVSQTESLKA AFITYLQAKQ AAGIINVPNP GSNQPAYVLQ IFP }\end{array}$

$\beta$

$\alpha$

$\beta$

$\beta$

C

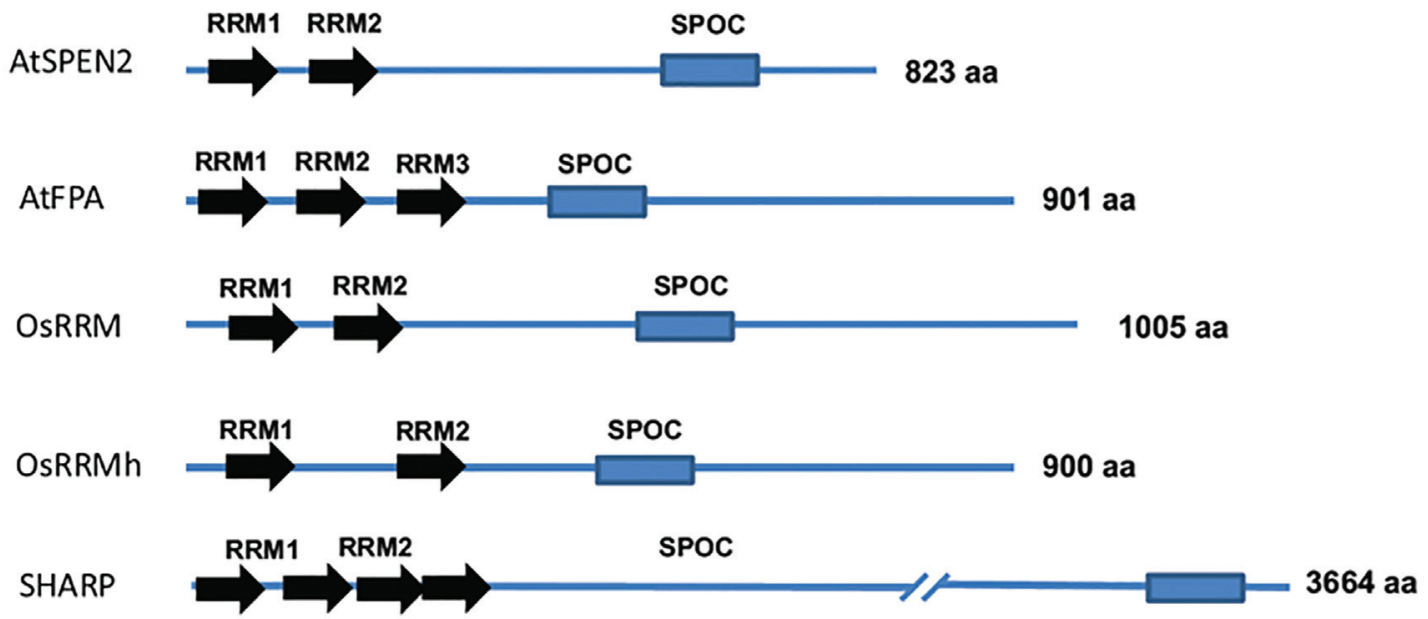

Figure 1 - The AtSpen 2 gene encodes a Spen putative protein. (A) The AtSpen2 gene is located on chromosome 4 from nucleotides 7,462,599 to $7,467,862$ in the $A$. thaliana genome (TAIR, The Arabidopsis Information Resource) and encodes an 823 aa putative protein, with two RRM domains (aa 25-89) and (aa 153-223), and a SPOC domain (aa 471-567) (http://www.ncbi.nlm.nih.gov/gene/826877). (B) Alignment of the SPOC sequences in Spen plant and human proteins: AtSPEN2 (A. thaliana), AtFPA (A. thaliana), OsRRM (O. sativa), OsRMMh $(O$. sativa) and SHARP (Homo sapiens) is shown. (C) Location of RRM and SPOC domains of Spen proteins: AtSPEN2 (A. thaliana), AtFPA (A. thaliana), OsRRM (O. sativa), OsRMMh (O. sativa) and SHARP (Homo sapiens).

There was no apparent difference among WT, KO and OE plants in any of the developmental traits analyzed: in Supplementary Figure S1, results of KO-E, OE-1, and WT, are shown as representatives; the other OE lines (OE-2 and OE-3) and the KO-P line, had statistically similar values in the phenotypic variables analyzed. These results discard the participation of the AtSpen 2 gene in the flowering time in the used experimental conditions, differently than its paralogue gene $f p a$.

The levels of AtSpen 2 transcripts in the OE-1 and KO-E lines used to analyze the phenotypic traits were determined by RT-qPCR. A. thaliana Col-0 plants were used 


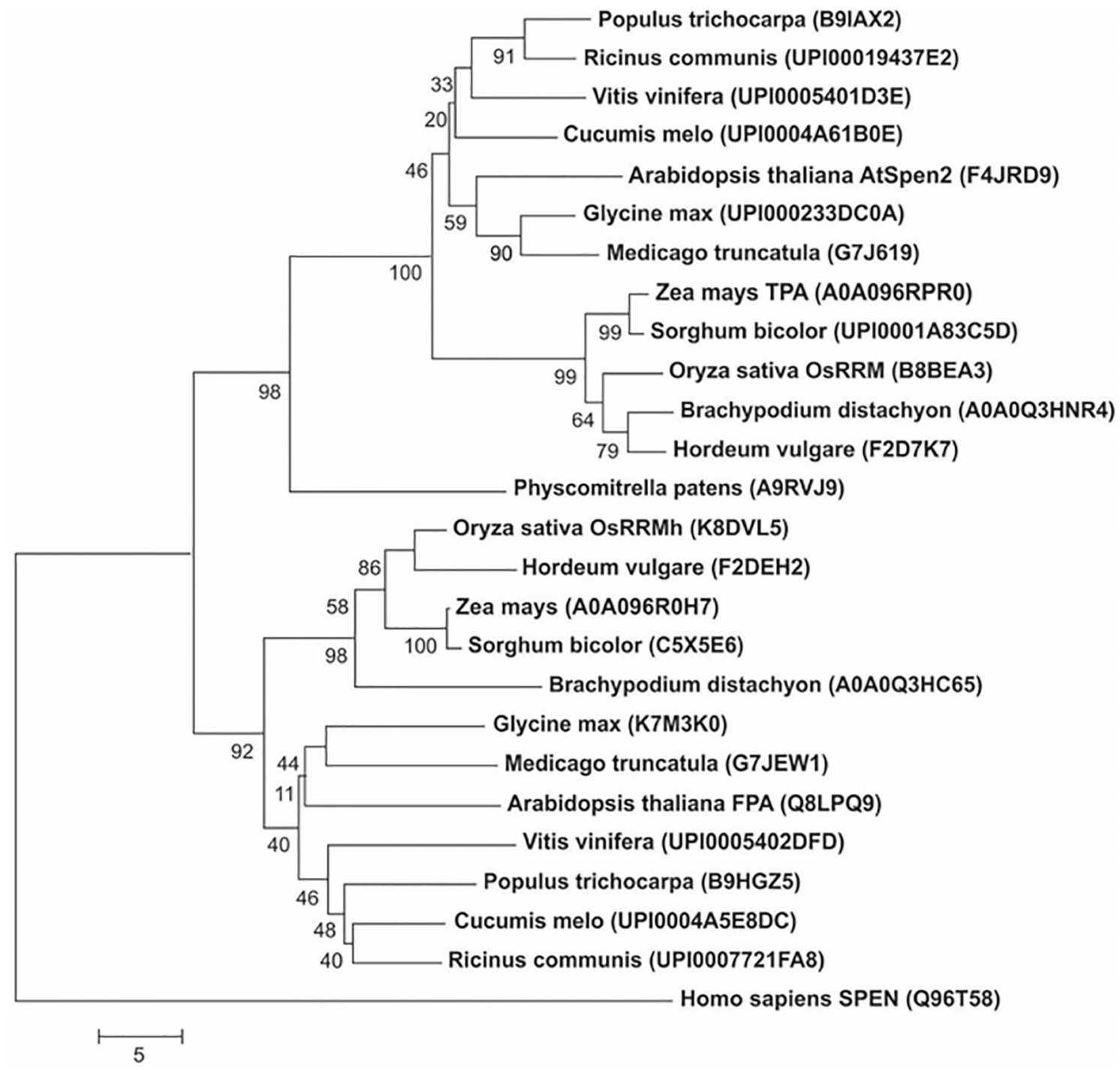

Figure 2 - Dendogram inferred using Neighbor-Joining of plant Spen proteins derived from the SPOC domain comparisons. The SPOC sequences of the following species were used: Arabidopsis thaliana, Populus trichocarpa, Vitis vinifera, Oryza sativa, Hordeum vulgare, Brachypodium distachyon, Physcomitrella patens, Cucumis melo, Medicago truncatula, Shorgum bicolor, Ricinus communis, Glicine max, Zea mays, and Homo sapiens as the external group. Bootstrap values are shown below the branches. The scale is in the units of the number of amino acid differences per sequence, and accession number or locus ID is indicated in parenthesis.

as the control, and the expression of the endogenous actin gene was used as the internal reference. In the different organs analyzed, leaf, root, and flower, there was greater expression of the AtSpen 2 gene in the OE-1 line (145.5, 29.5 and 41.2 fold, respectively), and lower in the KO-E line (0.011, 0.21 and 0.0023 fold, respectively), relative to WT seedlings (1, for all organs) (Figure 3 ). The changes observed in the AtSpen 2 transcript levels in the OE-1 line with respect to the WT plants were more than two magnitude orders in leaves and one magnitude order in root and flowers. In the KO-E line, changes in transcript levels were more variable, with decrements of two magnitude orders in leaves and flowers, but only five-fold in roots. Therefore, we consider that the observed changes in the AtSpen 2 transcript levels in OE-1 and KO-E lines with respect to WT

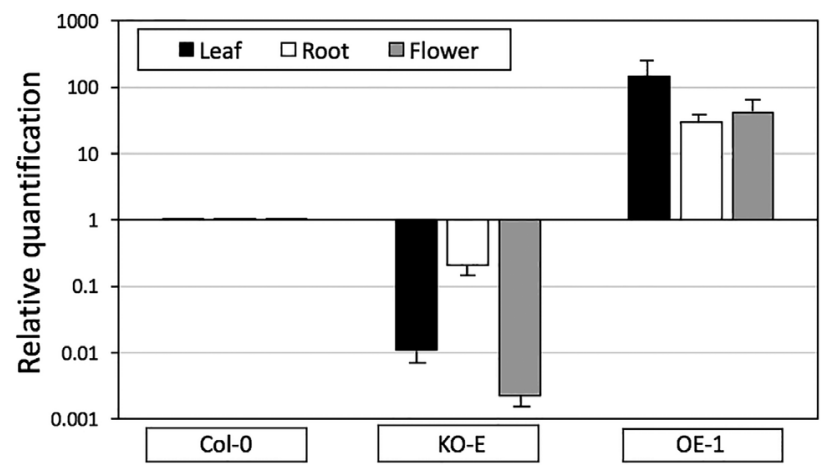

Figure 3 - AtSpen 2 expression levels in A. thaliana lines. AtSpen2 gene expression was determined by quantitative PCR, according to the Materials and Methods. Actin was used as the endogenous control. Expression in KO-E and OE-1 lines are reported as fold-change relative to the WT Col-0 line. 
plants are significantly high to be considered typical overexpressing and knockout lines. Furthermore, it is improbable that the KO-E line can produce a normal mRNA, because the exon 3 is interrupted by the T-DNA, as we demonstrated. Although only one endogenous gene was used as control, the experiment was repeated three times with similar results.

Cis elements and expression conferred by the AtSpen2 promoter to the uidA and gfp reporter genes

The promoter region of the AtSpen 2 gene apparently consists of $346 \mathrm{bp}$, which is the distance that separates it from the nearest gene, At4g12620. This promoter lacks a canonical TATA box, which is present in one-third of $A$. thaliana genes at $\sim 30$ bases upstream of the transcription start site; it also lacks the initiator element (Inr),
YYANa/tYY. Some potential cis-regulatory elements identified by bioinformatics tools (see Material and Methods) were T-Box (position 7462561-7462566), GATA-Box (position 7462503-7462508), and I-Box (position 7462504-7462509) (AGRIS: Davuluri et al., 2003 and Athena: O'Connor et al., 2005). The GATTTGATA sequence at position 746499-746507 was found, by visual analysis, as a variant of the GAaTTGATA and GAgaTGATA motifs, both reported cis-acting elements conferring expression in the phloem (Truernit and Sauer, 1995; Srivastava et al., 2014). This finding is congruent with the tissue-specific expression conferred by the AtSpen 2 promoter, as described in a subsequent section (Figure 4).

The promoter region of Atspen 2 is shared with the At4g12620 gene, which is located at position 74596467462253 (TAIR, The Arabidopsis Information Resource).

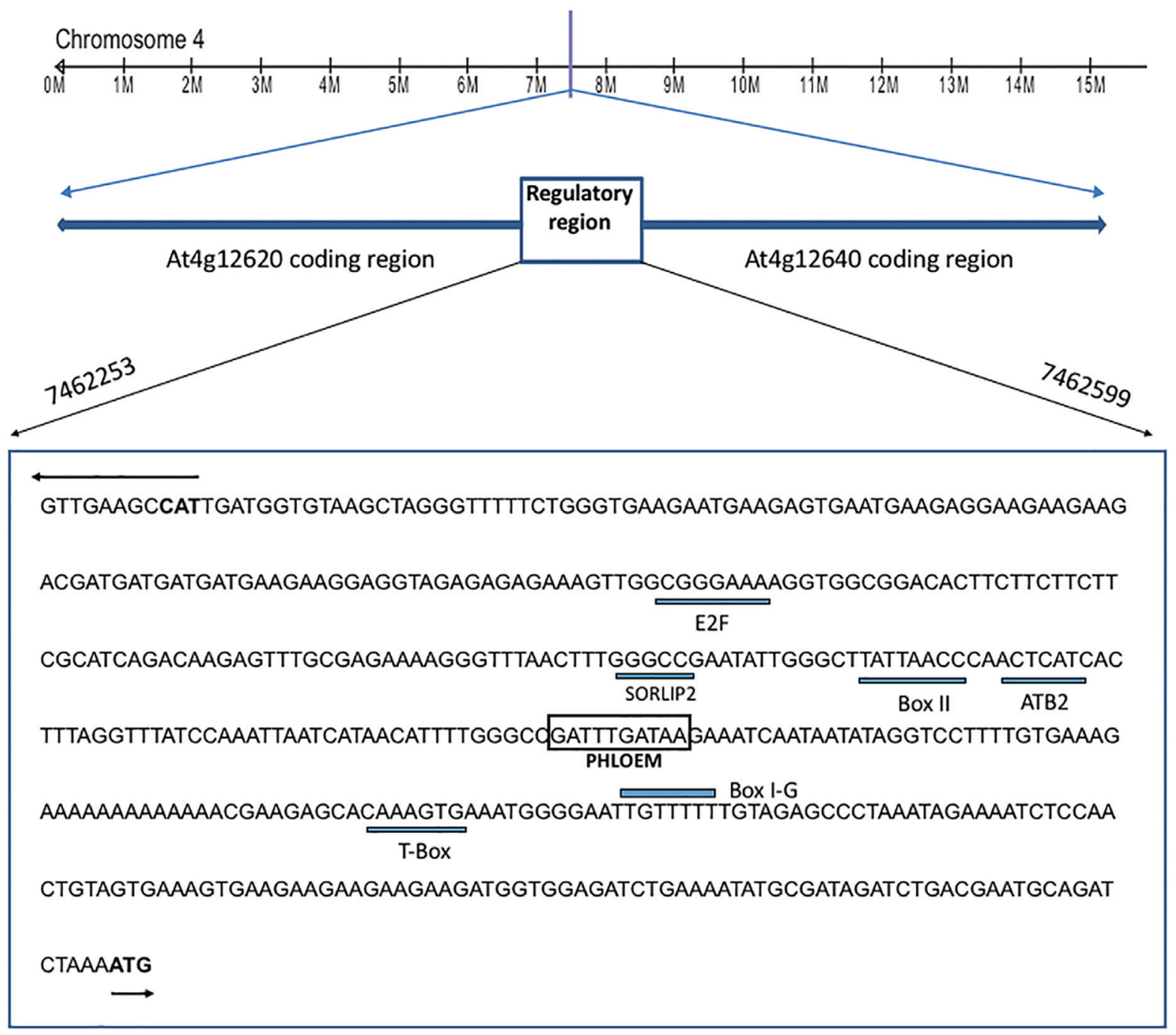

Figure 4 - Putative cis-regulatory elements in the AtSpen 2 promoter. The nucleotide sequence of 346 bp located 5' upstream of the AtSpen 2 coding sequence was used for the bioinformatics analysis; this sequence is partially shared with the promoter of the At4g12620 gene. The underlined sequences correspond to I-Box, GATA-Box and T-Box as part of the AtSpen2 promoter. The box corresponds to the putative element conferring expression in phloem. Other underlined sequences correspond to At4g12620 cis elements: T-Box, ATB2/AtbZIP53/GBF5 BS in ProDH, BoxII, SORLIP2 and E2F. 
This gene encodes a protein of the replication origin recognition complex (ORC1B) and its promoter region contains several predicted cis elements: T-Box, ATB2/AtbZIP53/GBF5 BS in ProDH, BoxII, SORLIP2 and E2F (AGRIS: Davuluri et al., 2003 and Athena: O'Connor et al., 2005) (Figure 4).

A. tumefaciens transformed with a binary vector harboring the AtSpen 2 promoter region fused to the uidA $:: g f p$ reporter genes was used to transform A. thaliana Col-0 plants. The transformed plants were differentiated from untransformed ones by their greenish color and fast growth in media with kanamycin $(60 \mu \mathrm{g} / \mathrm{mL})$. The percentage of plant transformation obtained was $1-1.2 \%$. Plants were selected for homozygosis in the T3 generation. Twelve independent transgenic lines were obtained, 10 of them with detectable expression of the two reporter genes, although with variations in the intensity of expression, possibly due to positional effects of the T-DNA insertion into the genome. The expression analysis of the reporter genes showed that both uidA and $g f p$ displayed the same expression patterns in the different plant tissues. Accordingly, the results illustrated in the subsequent figures are shown with either one or the other reporter gene, depending on the quality of the images obtained.

The expression of the uidA::gfp reporter genes was observed in different organs of the plants. In roots, the expression was found in the stele of the differentiated zone, both in the vascular tissue and pericycle. Lateral root primordia originating from pericycle showed expression in all cells, but in the tips of the primary and lateral roots, the expression was detected in the central part of the elongation and meristematic zones, and it was interrupted before reaching the quiescent center. (Figure 5A-D). In the cotyledonary and true leaves, the expression was restricted to the vascular tissue bundles and continued through the petiole (Figure 5E-H).

In the flowers, the expression was observed in the filaments of the stamens, but not in the anthers and pollen grains. Following the stages reported by Smyth et al. (1990), in flower stage 12 (flower unopened unfertilized), expression was concentrated in the septum margins (Figure 5I), whereas in the stages 14-15 (fertilized flowers), the expression appeared with greater intensity in the developing embryos (Figure 5J).

\section{Discussion}

In this work, the AtSpen 2 gene of A. thaliana and the predicted encoded protein were analyzed by using bioinformatics and experimental methods. The comparative sequence analysis showed that the putative AtSPEN2 protein is different from FPA, the first Spen protein functionally characterized in A. thaliana, in both size and overall amino acid sequence: AtSPEN2 exhibits two RRMs in its Nterminal domain whereas FPA displays three RRMs. Sys- tematic comparisons of the AtSPEN2 SPOC domain revealed that it exhibits higher sequence identity to the equivalent domain of Spen proteins encoded by other plant species, including the OsRRM protein of rice $(72 \%)$, whereas with $A$. thaliana FPA was only $56 \%$. Correspondingly, the SPOC domain of FPA exhibits higher sequence identity to the OsRRMh protein of rice than to AtSPEN2 (Figure 2). These observations indicate that the AtSpen 2 gene is a paralogue of the $f p a$ gene of $A$. thaliana, and probably an orthologue of the rice OsRRM gene. Thus, it is plausible that the AtSPEN2 protein function has diverged from that of FPA, which is involved in controlling flowering time by the autonomous pathway (Schomburg et al., 2001).

This hypothesis is supported by the results of the phenotypic analysis of $A$. thaliana AtSpen $2 \mathrm{KO}$ mutants and transgenic OE lines, as flowering time was not modified compared to WT plants. In contrast, inactivation of $f p a$ in A. thaliana (Schomburg et al., 2001) and its orthologous gene $O S R R M h$ in rice (Liu and Cai, 2013) produced a delay in flowering, hence demonstrating that the function of both proteins in flowering control has been preserved through the evolution of angiosperms. It can be hypothesized that the ancestral gene encoding a Spen protein in plants was duplicated before separation of dicots and monocots and that a process of functional divergence took place throughout the subsequent evolution of those plant lineages. The plausible original function of the ancestral protein was the control of flowering, as indicated by the preservation of that function in both FPA and OsRRMh, while the other proteins (AtSPEN2 and OsRRMh) might have one or more functions unrelated to this process.

We functionally analyzed the promoter of AtSpen 2 to establish its pattern of activity in different organs and tissues during plant development, and compared it with the expression conferred by the $f p a$ gene promoter (Figure 5, Table 1). This could shed light on potential functional differences between the Spen protein-coding genes examined because promoters have an important role in determining the place (tissue or organ), time, and intensity of transcription, which together with the characteristics of the encoded proteins, largely define the molecular, cellular, and physiological function of these proteins (Hernández-García and Finer, 2014). The absence of a canonical TATA box and an initiator element in the AtSpen 2 promoter indicates that these elements are not the only cis elements that are critical for determining transcription initiation of RNA pol II-dependent genes (Narlikar, 2014). Although it has been proposed that yeast and human genes with promoters lacking a TATA box are involved in constitutive processes (Yang et al., 2007), there are numerous exceptions to this rule in those same species and, notably, in plants (Kumari and Ware, 2013). Indeed, the pattern of tissue-specific expression conferred by the AtSpen 2 promoter and its dependence on the development of flower structures suggest 

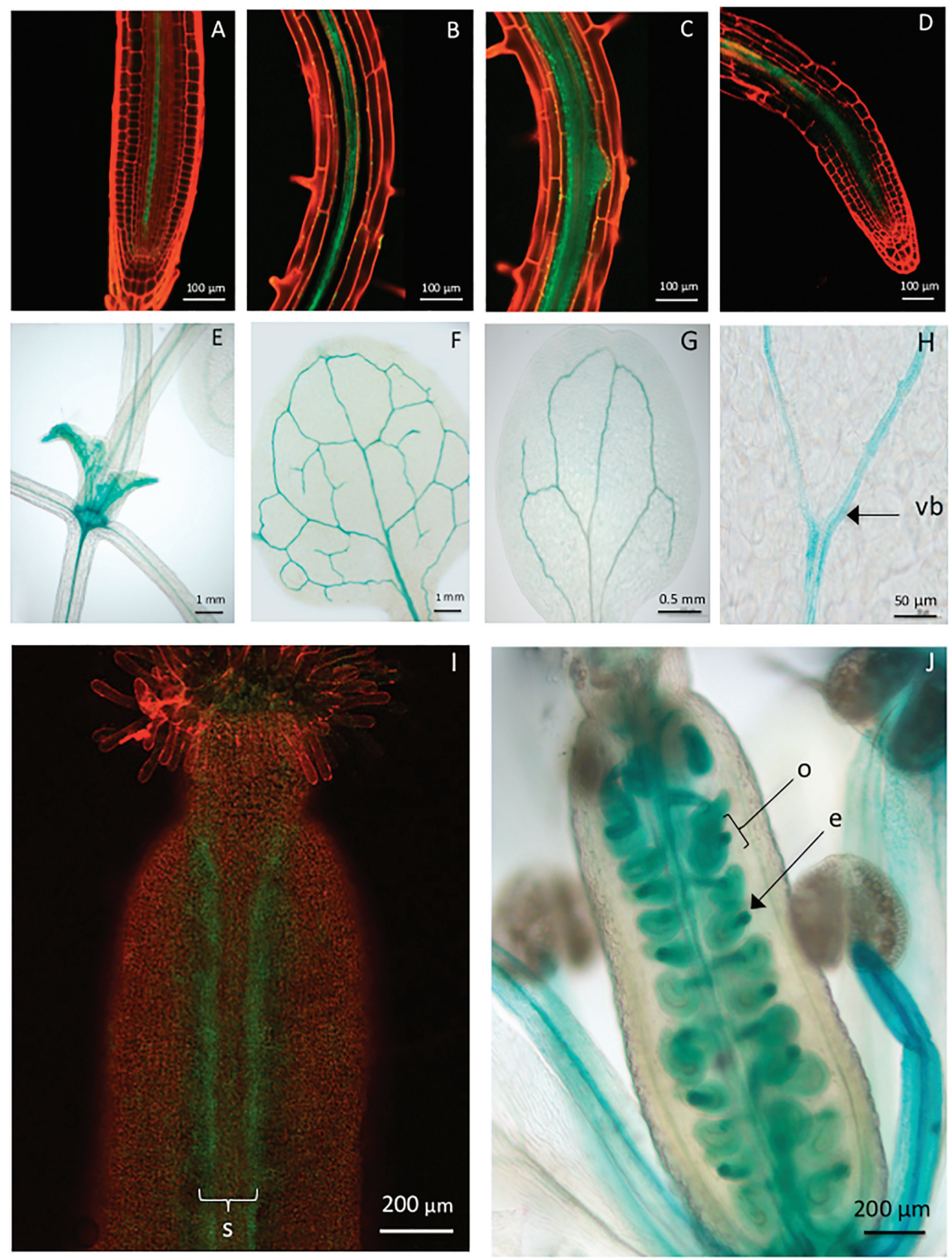

Figure 5 - Expression conferred by the AtSpen 2 promoter region in different organs and tissues. (A) Primary root tip; (B) lateral root tip; (C) lateral root primordium; (D) lateral root; (E) stem with forming leaves; (F) true leaf; (G) cotyledonary leaf; (H) vascular bundle; (I) gynoecium in the flower stage 13 (without fertilization); (J) gynoecium in the flower stage 15 (fertilized with developing embryos). In A-D and I, the expression was detected by GFP fluorescence (green). In E-H and J, the expression was detected by the uidA reporter gene (blue). Images are representative of 10 replicates. vb, vascular bundle; s, septum; o, ovule; e, embryo. 
Table 1 - Comparison of organ and tissue-specific expression patterns of the uidA reporter gene directed by the AtSpen 2 and fpa gene promoters in Arabidopsis thaliana (Data of the fpa promoter are those reported by Schomburg et al., 2001).

\begin{tabular}{|c|c|c|}
\hline ORGANS/ TISSUES & fpa EXPRESSION & AtSpen 2 EXPRESSION \\
\hline COTYLEDONARY LEAVES & $\begin{array}{l}\text { Yes } \\
\text { Pattern: the whole leaf }\end{array}$ & $\begin{array}{l}\text { Yes } \\
\text { Pattern: vascular tissue }\end{array}$ \\
\hline ROOTS & No & $\begin{array}{l}\text { Yes } \\
\text { Pattern: all the stele of primary and lateral roots (vascu- } \\
\text { lar tissue and pericycle); lateral root primordia } \\
\text { Time: all time }\end{array}$ \\
\hline MATURE LEAVES & $\begin{array}{l}\text { Yes } \\
\text { Pattern: patches } \\
\text { Time: only in the two first true leaves }\end{array}$ & $\begin{array}{l}\text { Yes } \\
\text { Pattern: vascular tissue } \\
\text { Time: all time }\end{array}$ \\
\hline AXILARY MERISTEM INFLORESCENCE & $\begin{array}{l}\text { Yes } \\
\text { Pattern: homogeneous }\end{array}$ & No \\
\hline INFLORESCENCE STEM & $\begin{array}{l}\text { Yes } \\
\text { Pattern: homogeneous }\end{array}$ & $\begin{array}{l}\text { Yes } \\
\text { Pattern: vascular tissue }\end{array}$ \\
\hline FLOWER & $\begin{array}{l}\text { Yes Pattern: not reported } \\
\text { Time: all time }\end{array}$ & $\begin{array}{l}\text { Yes } \\
\text { Pattern: septum in stage } 12 \text { (non-fertilized flower); de- } \\
\text { veloping embryos in stages 14-15. } \\
\text { Time: onset at stage } 12\end{array}$ \\
\hline
\end{tabular}

that this promoter does not display constitutive transcriptional activity.

The most characteristic expression directed by the AtSpen 2 promoter occurs throughout the vascular tissue (phloem and xylem) and although some cis elements have been associated to the specific expression in phloem, the reported sequences are diverse, consisting of 8-13 nucleotides that do not show a significant degree of conservation among distinct promoters. These phloem-specific sequences have been classified into four groups, two of which have been reported in A. thaliana (Srivastava et al., 2014). The sequence GATTTGATA found in the AtSpen 2 promoter is very similar to the sequences GAaTTGATA and GAgaTGATA, both reported to confer expression in Pisum sativum (Srivastava et al., 2014) and A. thaliana (Truernit and Sauer, 1995). Thus, it is possible that this sequence is involved in the phloem expression conferred by the AtSpen 2 promoter. Another possibility is the existence of a different cis element (or combination of them) in the AtSpen 2 promoter conferring expression in phloem, but delimiting it needs another approach, such as directed mutagenesis.

Although the reporter gene expression directed by the AtSpen 2 promoter was observed throughout the vascular tissue, it is not clear what function it could have in this tissue, unlike other $A$. thaliana genes that are expressed in the phloem, such as $A H A 3$ and $S U C 2$. The former gene encodes a $\mathrm{H}^{+}$-ATPase, which provides energy for SUC2, a sucrose- $\mathrm{H}^{+}$symporter, both contributing to the transport of sucrose in this tissue (Truernit and Sauer, 1995). Another relevant example is the Cucurbita moschata PP2 gene encoding one of the major proteins involved in the formation of slime plugs in damaged phloem (Guo et al., 2004). While the reported Spen proteins participate in regulating the expression of other genes in animals (Sánchez-Pulido et al., 2004; McHugh et al., 2015) and plants such as FPA, which regulates the expression of the flowering repressor FLC (Hornyik et al., 2010), it is possible that AtSPEN2 contributes to the regulation of a gene whose protein could have a clear function in the phloem.

The root is an organ that allows the plant to be anchored to the substrate and to absorb water and nutrients from the soil and transport them to the aerial parts via the vascular tissue, which is a central stele in A. thaliana, both in the primary and lateral roots (Scheres et al., 2002). The proposal that AtSpen 2 function is partly associated with the vascular tissue is reinforced by the expression conferred by the promoter in this tissue, through the root system and the aerial parts, indicating that this gene is being expressed throughout all of the plant vascular system. In the root, the expression of AtSpen 2 extends to the pericycle cell layer, the site where lateral roots originate through robust hormonal and environmental control (Dubrovsky et al., 2008). In transformed fpa::uidA plants, expression in the root is not reported (Schomburg et al., 2001, Table1), adding another important difference between fpa and AtSpen 2 and reinforcing the notion of a functional difference between these two genes and their encoded proteins. Besides, in our experimental analysis, the expression conferred by the AtSpen 2 promoter in leaves is restricted to the vascular tissue. In contrast, the $f p a$ promoter region confers expression mainly in young leaves and in continuous foliar areas that are not associated with the vascular tissue (Schomburg et al., 2001, Table 1), which is an important difference that supports the divergence of the function of both genes in $A$. thaliana.

The expression conferred by the AtSpen 2 promoter in the ovules and developing embryos suggests that this gene plays a role in this stage, but additional studies are required to confirm this because it has been reported that embryo- 
genesis is complex and involves the acquisition of cellular identities and developmental patterns (Capron et al., 2009). Although the fpa gene is also expressed in flowers, its pattern is different from that conferred by the AtSpen 2 promoter, because this expression is widely spread throughout the inflorescence and begins in the initial stages of flower development (Schomburg et al., 2001), which is consistent with the function of this gene in the control of flowering time. A comparison of the expression time of the $A$. thaliana and $O$. sativa genes encoding Spen proteins in the developing of the reproductive structures reinforce the hypothesis of divergence of fpa and Atspen 2 functions. In the rice genes $O s R R M h$ and $O s R R M$ (orthologous of fpa and AtSpen 2, respectively), the expression in the former (determined by RT-qPCR) occurs in the leaves and inflorescences (Liu and Cai, 2013), while the OsRRM expression (determined by a reporter gene) is associated only with the seed endosperm (Chen et al., 2007). Summarizing this comparison, the expression in the flower of the orthologous genes fpa and $O s R R M h$ is found at an early flowering stage, in congruence with their reported function in controlling the flowering time, while the orthologous genes AtSpen 2 and $O s R R M$ are expressed only in later flower stages.

Although the AtSpen 2 promoter directed reporter gene expression in the transition from the unfertilized flower stage to the flower with developing embryos, we did not observe any changes in the floral development of the plant when the AtSpen 2 gene was inactivated or overexpressed. Some authors have reported that in consequence of gene inactivation there may not necessarily be a morphological change, even if there is a severe physiological alteration; consequently, integral strategies to reveal the function of these genes should be used (Niehaus et al., 2015). The redundancy in the function of genes may account for the absence of a phenotype when a gene is mutated (Bouché and Bouchez, 2001), but in the particular case of AtSpen2, this is unlikely because of the following reasons: a) its unique paralogue in $A$. thaliana, the gene $f p a$, has a function in the control of flowering time (Ietswaart et al., 2012), which was not found for AtSpen2 in this work; b) the SPOC domains of FPA and AtSPEN2 have low sequence identity; and c) the expression conferred by the promoter regions of AtSpen 2 and fpa in the organs and tissues of A. thaliana, respectively, is completely different. Therefore, it is proposed that the lack of a remarkable phenotype in our $\mathrm{KO}$ and OE lines is due to any of the following situations: a) AtSpen 2 is not a master gene in a developmental process, but it may have an accessory function, and b) its participation is conditional and depends on certain endogenous or environmental conditions.

\section{Conclusions}

Taken together, our results demonstrate that the AtSpen 2 gene: a) has a functional promoter that confers expression in the whole vascular tissue, and in the gynoecium and developing embryos, and b) its function in the regulation of the flowering time was discarded, and this is due possibly to the divergence of the transcription expression pattern and low similarity of the SPOC domain with its paralogue fpa gene.

\section{Acknowledgments}

We thank the Coordinación General de Estudios de Posgrado of the Universidad Michoacana by a grant to María Gloria Solís-Guzmán, and to CONACYT for the support of project CB-2011-169769.

\section{References}

Alonso JM, Stepanova AN, Leisse TJ, Kim CJ, Chen H, Shinn P, Stevenson DK, Zimmerman J, Barajas P, Cheuk R, et al. (2003) Genome-wide insertional mutagenesis of Arabidopsis thaliana. Science 301:653-657.

Arabidopsis Genome Initiative (2000) Analysis of the genome sequence of the flowering plant Arabidopsis thaliana. Nature 408:796-815.

Ariyoshi M and Schwabe JW (2003) A conserved structural motif reveals the essential transcriptional repression function of Spen proteins and their role in developmental signaling. Genes Dev 17:1909-1920.

Artavanis-Tsakonas S, Rand MD and Lake RJ (1999) Notch signaling: Cell fate control and signal integration in development. Science 284:770-776.

Bouché N and Bouchez D (2001) Arabidopsis gene knockout: Phenotypes wanted. Curr Opin Plant Biol 4:111-117.

Capron A, Chatfield S, Provart N and Berletha T (2009) Embryogenesis: Pattern formation from a single cell. The Arabidopsis Book 7:e0126.

Chen F and Rebay I (2000) Split ends, a new component of the Drosophila EGF receptor pathway, regulates development of midline glial cells. Curr Biol 10:943-946.

Chen SY, Wang ZY and Cai XL (2007) OsRRM, a Spen-like rice gene expressed specifically in the endosperm. Cell Res 17:713-721.

Clough SJ and Bent AF (1998) Floral dip: A simplified method for Agrobacterium-mediated transformation of Arabidopsis thaliana. Plant J 16:735-43.

Davuluri RV, Sun H, Palaniswamy SK, Matthews N, Molina C, Kurtz M and Grotewold E (2003) AGRIS: Arabidopsis Gene Regulatory Information Server, an information resource of Arabidopsis cis-regulatory elements and transcription factors. BMC Bioinformatics 4:25.

Dubrovsky JG, Sauer M, Napsucialy-Mendivil S, Ivanchenko MG, Friml J, Shishkova S, Celenza J and Benkova E (2008). Auxin acts as a local morphogenetic trigger to specify lateral root founder cells. Proc Natl Acad Sci U S A 105:87908794.

Guo H, Cheny X, Zhang H, Fang R, Yuan Z, Zhang Z and Tian Y (2004) Characterization and activity enhancement of the phloem-specific pumpkin PP2 gene promoter. Transgenic Res 13:559-566.

Haseloff J and Amos B (1995) GFP in plants. Trends Genet 11:328-329.

Hepworth SR, Valverde F, Ravenscroft D, Mouradov A and Coupland G (2002) Antagonistic regulation of flowering- 
time gene SOC1 by CONSTANS and FLC via separate promoter motifs. EMBO J 21:4327-4337.

Hernández-Garcia CM and Finer JJ (2014) Identification and validation of promoters and cis-acting regulatory elements. Plant Sci 217:109-119.

Hornyik C, Terzi LI and Simpson GG (2010) The Spen family protein FPA controls alternative cleavage and polyadenylation of RNA. Dev Cell 18:203-213.

Ietswaart R, Wu Z and Dean C (2012) Flowering time control: Another window to the connection between antisense RNA and chromatin. Trends Genet 28:445-453.

Jefferson RA, Kavanagh TA and Bevan MW (1987). GUS fusions: $\beta$-glucuronidase as a sensitive and versatile gene fusion marker in higher plants. EMBO J 6:3901-3907.

Kuang B, Wu SCY, Shin YA, Luo L and Kolodziej P (2000) Split ends encodes large nuclear proteins that regulate neuronal cell fate and axon extension in the Drosophila embryo. Development 127:1517-1529.

Kumari S and Ware D (2013) Genome-wide computational prediction and analysis of core promoter elements across plant monocots and dicots. PLoS One 8:e79011.

Lane ME, Elend M, Heidmann D, Herr A, Marzodko S, Herzig A and Lehner CF (2000) A screen for modifiers of cyclin E function in Drosophila melanogaster identifies Cdk2 mutations, revealing the insignificance of putative phosphorylation sites in Cdk2. Genetics 155:233-244.

Liu F, Marquardt S, lister C, Swiezewski S and Dean C (2010) Targeted 3' processing of antisense transcripts triggers Arabidopsis FLC chromatin silencing. Science 327:94-97.

Liu D and Cai X (2013) OsRRMh, a Spen-like gene, plays an important role during the vegetative to reproductive transition in rice. J Integr Plant Biol 55:876-887.

Malamy JE and Benfey PN (1997) Organization and cell differentiation in lateral roots of Arabidopsis thaliana. Development 124:33-44.

Marchler-Bauer A, Derbyshir MK, Gonzales NR, Lu S, Chitsaz F, Geer LY, Geer RC, He J, Gwadz M, Hurwitz DI, et al. (2015) CDD: NCBI's conserved domain database. Nucleic Acids Res 43:D222-D226.

Maris C, Dominguez C and Allain FH (2005) The RNA recognition motif, a plastic RNA-binding platform to regulate posttranscriptional gene expression. FEBS J 272:2118-2131.

Martínez M (2011) Plant protein-coding gene families: Emerging bioinformatics approaches. Trends Plant Sci 16:558-567.

Martínez-Trujillo M, Limones-Briones V, Cabrera-Ponce JL and Herrera-Estrella L (2004) Improving transformation efficiency of Arabidopsis thaliana by modifying the floral dip method. Plant Mol Biol Rep 22:63-70.

McBride KE and Summerfelt KR (1990) Improved binary vectors for Agrobacterium-mediated plant transformation. Plant Mol Biol 14:269-276.

McHugh CA, Chen CK, Chow A, Surka CF, Tran C, McDonel P, Pandya-Jones A, Blanco M, Burghard C, Moradian A, et al. (2015) The Xist IncRNA interacts directly with SHARP silence transcription through HDAC3. Nature 521:232-236.

Murashige T and Skoog F (1962) A revised medium for rapid growth and bioassays with tobacco tissue culture. Physiol Plantarum 15:473-497.

Narlikar L (2014) Multiple novel promoter-architectures revealed by decoding the hidden heterogeneity within the genome. Nucleic Acids Res 42:12388-12403.
Niehaus TD, Thamm AMK, Crécy-Lagard V and Hanson AD (2015) Proteins of unknown biochemical function: A persistent problem and a roadmap to help overcome it. Plant Physiol 169:1436-1442.

O'Connor TR, Dyreson C and Wyrick JJ (2005) Athena: A resource for rapid visualization and systematic analysis of Arabidopsis promoter sequences. Bioinformatics 21:4411$-4413$.

Odell JT, Nagy F and Chua NH (1985) Identification of DNA sequences required for activity of the cauliflower mosaic virus 35S promoter. Nature 313:810-812.

Oswald F, Kostezka U, Astrahantseff K, Bourteele S, Dillinger K, Zechner U, Ludwig L, Wilda M, Hameister H, Knöchel W, et al. (2002) SHARP is a novel component of the Notch/RBP-Jk in the signaling pathway. EMBO J 21:5417-5426.

Rhee SY and Mutwil M (2014) Towards revealing the functions of all genes in plants. Trends Plant Sci 19:212-221.

Sammut SJ, Finn RD and Bateman A (2008). Pfam10 years on: 10 000 families and still growing. Brief Bioinform 9:210-219.

Sánchez-Pulido L, Rojas AM, van Wely KH, Martinez-A C and Valencia A (2004) SPOC: A widely distributed domain associated with cancer, apoptosis and transcription. BMC Bioinformatics 5:91.

Sanger F, Nicklen S and Coulson AR (1977) DNA sequencing with chain-terminating inhibitors. Proc Natl Acad Sci U S A 74:5463-5467.

Scheres B, Benfey P and Dolan L (2002) Root development. Arabidopsis Book 1:e0101.

Schomburg FM, Patton DA, Meinke DW and Amasino RM (2001) FPA, a gene involved in floral induction in Arabidopsis, encodes a protein containing RNA-recognition motifs. Plant Cell 13:1427-1436.

Simpson DD and Dean C (2002) Arabidopsis, the rosetta stone of flowering time? Science 296:285-289.

Simpson DD (2004) The autonomous pathway: Epigenetic and post-transcriptional gene regulation in the control of Arabidopsis flowering time. Curr Opin Plant Biol 7:570574.

Smyth DR, Bownan JL and Meyerowitz EM (1990) Early flower development in Arabidopsis. Plant Cell 2:755-767.

Srivastava VK, Raikwar S and Tuteja N (2014) Cloning and functional characterization of the promoter of PSSEOF1 gene from Pisum sativum under different stress conditions using Agrobacterium-mediated transient assay. Plant Signal Behav 9:e29626.

Tamura K, Stecher G, Peterson D, Filipski A and Kumar S (2013) MEGA6: Molecular Evolutionary Genetics Analysis version 6.0. Mol Biol Evol 30:2725-2729.

Truernit E and Sauer N (1995) The promoter of the Arabidopsis thaliana $\mathrm{SUC} 2$ sucrose-H+ symporter gene directs expression of beta-glucuronidase to the phloem: Evidence for phloem loading and unloading by SUC2. Planta 196:564-570.

Wiellette EL, Harding KW, Mace KA, Ronshaugen MR, Wang FY and McGinnis W (1999) Spen encodes an RNP motif protein that interacts with Hox to repress the development of head-like sclerites in the Drosophila trunk. Development 126:5373-5385.

Yang C, Bolotin E, Jiang T, Sladek FM and Martinez E (2007) Prevalence of the initiator over the TATA box in human and 
yeast genes and identification of DNA motifs enriched in human TATA-less core promoters. Gene 389:52-65.

Zhang Y, Rataj K, Simpson GG and Tong L (2016) Crystal structure of the SPOC domain of the Arabidopsis flowering regulator FPA. PLoS One 11:0160694.

\section{Internet Resources}

The databases and bioinformatics tools used in this study are available at the following electronic addresses:

Genetic analysis and molecular biology in Arabidopsis thaliana: TAIR The Arabidopsis Information Resource, www.arabidopsis.org

Analysis of promoter regions and transcription factors of Arabidopsis

thaliana: http://arabidopsis.med.ohio-state.edu/

Analysis of nucleotide and protein sequences:

http://www.ncbi.nlm.nih.gov/

http://www.sanger.ac.uk/ http://www.genomesonline.org

Analysis of proteins and domains:

http://pfam.sanger.ac.uk/

http://pfam.janelia.org/

http://pfam.sbc.su.se/

http://www.ncbi.nlm.nih.gov/entrez/query.fcgi? $d b=c d d$

http://www.uniprot.org

http://www.expasy.org/prosite/

\section{Supplementary material}

The following online material is available for this article: Figure S1 - Analysis of growth and development in $A$. thaliana lines.

Associate Editor: Marcia Pinheiro Margis

License information: This is an open-access article distributed under the terms of the Creative Commons Attribution License (type CC-BY), which permits unrestricted use, distribution and reproduction in any medium, provided the original article is properly cited. 